\title{
Design of Angle Measurement System Based on CPLD and MSP430
}

\author{
Wei Wang ${ }^{1, a}$, Liping Zheng ${ }^{2, b}$ \\ ${ }^{1}$ Department 1, Mechanical Engineering College, Shijiazhuang 050003, China; \\ 2 Department 1, Mechanical Engineering College, Shijiazhuang 050003, China. \\ a675654783@qq.com, b13191885181@163.com
}

Keywords: MSP430, CPLD, grating signal, angle measurement.

\begin{abstract}
In order to solve the problem of wide-range and high-precision angle measurement, a kind of grating-angle measurement system was designed. The grating sensor was used as output part of angle measurement signal, and the grating signal hardware decoding programme was designed applying CPLD, which achieved signal division and direction discerning and counting. The MSP430 MCU was used as control unit to achieve angle number calculation and display, communicating with CPLD by parallel bus. The design was verified its actual working effect by experiment tests, and the experiment data was finally analysized. The result showed that the system could reach $0.25 \mathrm{mil}$ measuring accuracy, working stably with quality of low-power and high-precision.
\end{abstract}

\section{Introduction}

Angle measurement is one of common problems in engineering practice, which has numerous measurement methods, and the grating measurement method is widely used at present with quality of high precision and large range ${ }^{[1]}$. The grating measuring method applied Moire fringe principle, converting angular displacement to voltage signal orthogonal characteristics, and could further improve the measurement resolution through high-power electronic subdivision, whose measuring and decoding forms were flexible, very suitable for engineering measurement ${ }^{[2]}$.

In this paper, a kind of grating angle measurement system was designed using CPLD and MSP430 MCU as control chip. The MCU owns quality of fast processing and ultra-low power consumption, can effectively regulate the balance of system performance and power consumption; The CPLD circuit can be programmed, could parallel process signal with high-speed, which is easy to facilitate the maintenance and debug. Compared with the single MCU or CPLD as the core of the measurement system, the combined design can give full play to the ultra-low power characteristics of MSP430 MCU and CPLD high speed processing performance with greater flexibility.

\section{Overall Design}

The system was composed of arc-circular grating angle sensor, signal processing unit and peripheral devices. The angle sensor induced angle changes and generated grating measurement signal, the CPLD chip dealt with signal by distinguishing counting operation, as the control unit, MSP430 MCU controlled CPLD decoding function and data parallel communication, as well as completing number calculation and LCD display; The keyboard was used as input device, and the power provided same reference voltage for entire system, the whole structure was shown in figure 1.

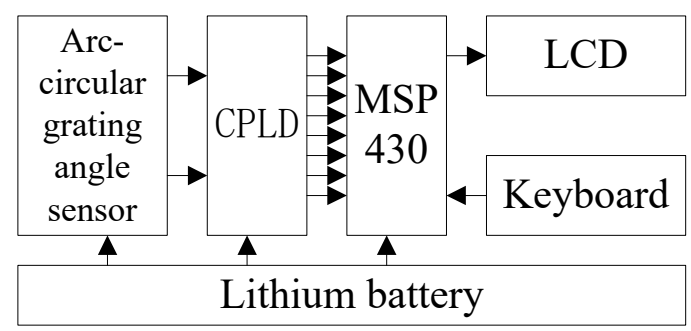

Fig.1 System structure 


\section{Design of Hardware Section}

Angle Sensor. As the signal input part of the measurement system, the quality of the signal determines the reliability of the measurement results, which directly affects the accuracy of the angle measurement. System applied the sensor which obtained national invention patent, and the structure was shown in figure 2.

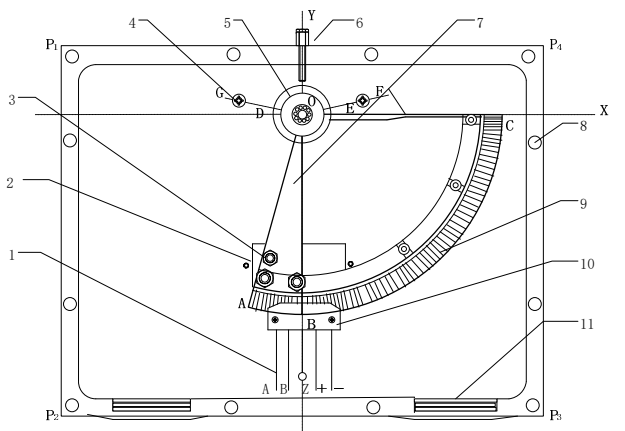

Fig.2 Structure of Arc-grating Angle Sensor

Each part in the figure: 1power line, 2gravity calibration window, 3 weight limit screw nut, 4limit screw, 5laps, 6screw plug, 7main arm swing grating, 8screw holes, 9moving scale grating, 10reading head grating, 11 magnet.

The sensor work through grating pendulum, measuring range from $-20^{\circ}$ to $90^{\circ}$, the grating line destiny was 6000/round, and the resolution was $1 \mathrm{mil}$. The sensor contained signal conditioning circuit, which aiming at amplifying and filtering the original signal, the final output signal was two orthogonal square wave whose phase difference was 90 degree, and the measurement resolution could reach $0.25 \mathrm{mil}$ after four times of electronic subdivision ${ }^{[3]}$. The working voltage of the sensor was $5 \mathrm{~V}$, the high level pulse of the output grating signal was about $3.3 \mathrm{~V}$, the low level was $0 \mathrm{~V}-0.1 \mathrm{~V}$, which was in accordance with the requirements of TTL logic level, and could directly enter the CPLD processing chip. The signal waveform was shown in figure 3.

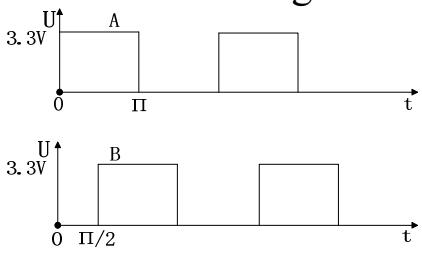

Fig.3 Grating signal waveform

Mainboard circuit. Considering the design cost and power consumption, the EPM240T100C5 CPLD chip of Altera company was selected as decoding unit, the MSP430F6638 MCU was selected as control unit. The EPM240 chip was provided with $50 \mathrm{MHz}$ frequency crystal oscillator and MSP430F6638 with 32KHz, the CPLD reset pin was controlled by MCU, parallel output 16bit data to $\mathrm{MCU}$, and the LCD was connected to the internal drive special interface. The polymer lithium battery was used as power to supply the system through SPX3819 voltage conversion circuit, the circuit was as shown in figure 4.

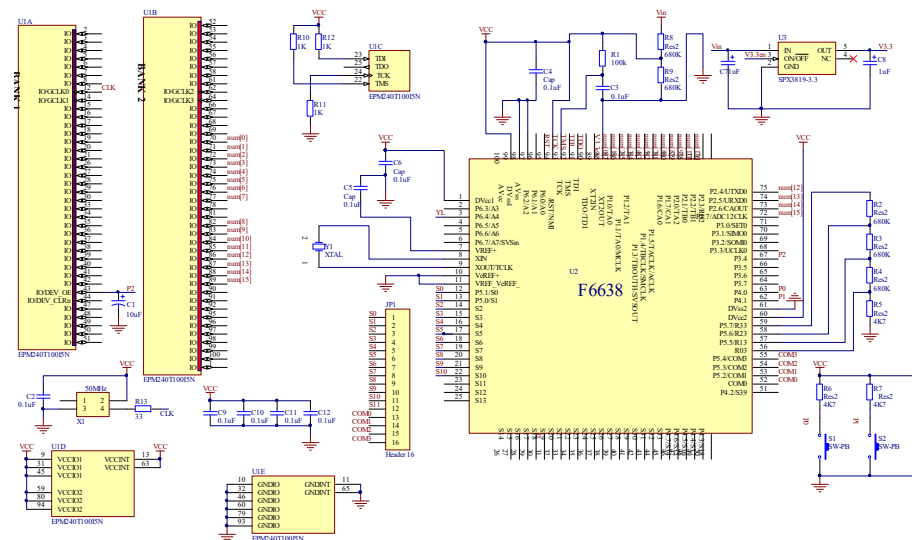

Fig. 4 Chip circuit 
Peripheral devices. Peripheral devices consisted of keyboard and LCD. The keyboard was used to realize the control function of MCU, including measurement switch and numerical reset. LCD display cache could be updated directly by MCU.

\section{Design of Software Section}

\section{CPLD Program}

Principle of Signal Subdivision and Direction Distinguishing. The output signal A and B from sensor have four level states of $00,10,11,01$ in the same cycle, which maintained T/4 for each state, and this is the four times subdivision principle. When the sensor deflected, grating moved forward, the variety rule of level are as follows:(AB) 10-11-01-00-10; if reverse motion, the variety rule of level are as follows: (AB)10-00-01-11-10. According to the motion of signal, the deflection direction of the angle of inclination is judged ${ }^{[4]}$.

Module Program. The program comprised module of subdivision and counting, each module took the rising edge of the clock signal and the falling edge of the reset signal as trigger condition, which executed program parallel. Set variable temp and temp1, temp1 read signal level of A and B on each rising edge of clock signal, temp read value of temp1 with non-blocking assignment way, when the signal level changes, we can determine the movement signal through comparing the value of temp and temp1. Set variable num1 and num2, respectively on behalf of the positive counter register and reverse counter register, when the signal move forward, variable num1 add 1; when the signal move reverse, variable num 2 minus 1 . Variable num represented the final result, obtained by subtraction operation of num 1 and num2. The program run as shown in figure 5.
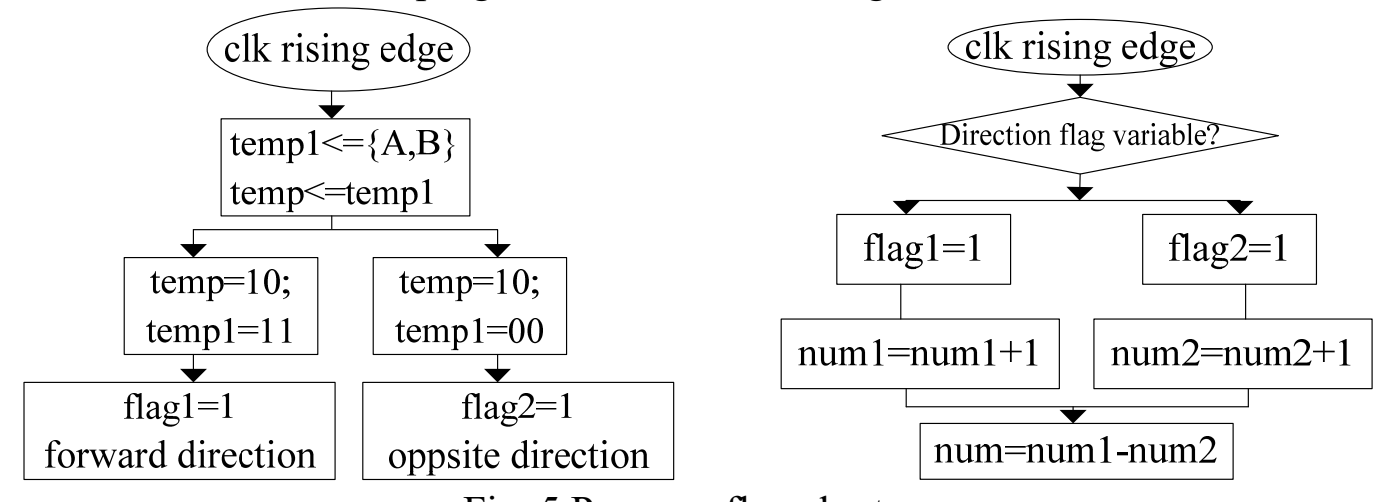

Fig. 5 Program flowchart

In the figure, flag1 and flag2 were used as direction sign variable, respectively indicating the forward motion state and the reverse motion state.

\section{MSP430 Program}

Taking IAR software of Embedded Workbench as MSP430 integrated developing environment, the program consisted of initial part and interruption part, according to ultra-low power design law ${ }^{[5]}$. The initialization procedure executed module setting of clock system, the I/O port, the timer and so on, and the interrupt procedure executed LCD display program, updating the measurement result. The program flow chart was shown in Figure 6.
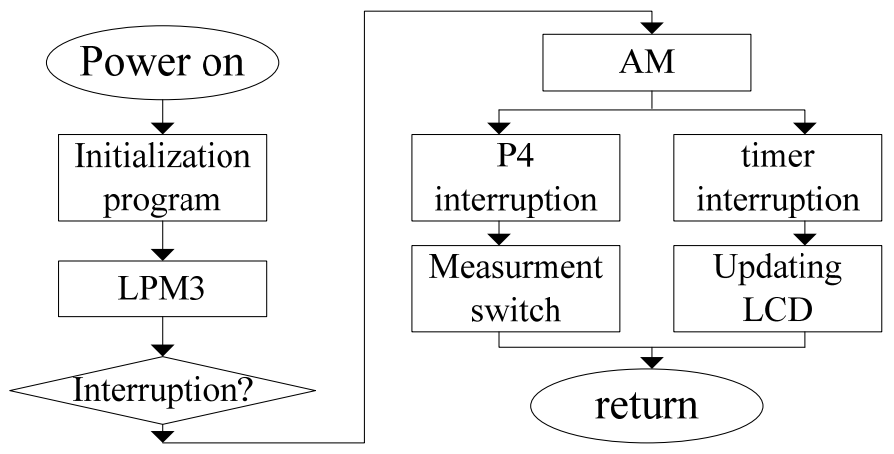

Fig. 6 Program flowchart 


\section{Experiment}

The system was placed on the axis gyro instrument to test, the measuring process was as follows: The system was placed on the axis gyro instrument to test, the measuring process is as follows: firstly leveling the platform, and then turn the experimental station, Measuring once per interval 100mil, according to the close relationship with the degree of conversion: $1 \mathrm{mil}=0.06^{\circ}$, record measurement data per interval $6^{\circ}$; When the turntable reached $18^{\circ}$, turn reverse rotation until the platform leveling again. In order to ensure the reliability of the measurement, the data were tested for three times and the results were shown in Table 1.

Table 1 Testing results

\begin{tabular}{cccccc}
\hline Numble & Indication & Value 1 & Value 2 & Value 3 & Maxmum error \\
\hline 1 & $0.000^{\circ}$ & 0.00 & 0.00 & 0.00 & 0.00 \\
2 & $6.000^{\circ}$ & 100.00 & 100.25 & 100.00 & +0.25 \\
3 & $12.000^{\circ}$ & 199.75 & 200.00 & 199.75 & -0.25 \\
4 & $18.000^{\circ}$ & 299.75 & 299.75 & 299.75 & -0.25 \\
5 & $12.000^{\circ}$ & 199.75 & 200.25 & 199.75 & +0.25 \\
6 & $6.000^{\circ}$ & 100.00 & 100.00 & 100.00 & 0.00 \\
7 & $0.000^{\circ}$ & 0.25 & 0.00 & -0.25 & -0.25 \\
\hline
\end{tabular}

Unit: mil

As shown in table 1, The system measuring results were essentially in agreement with indication of experimental station, take maximum error value as parameter, and calculate the average value of absolute maximum error value, the calculation formula was as follows:

$$
\mu=\frac{\left|x_{1}\right|+\left|x_{2}\right|+\cdots+\left|x_{11}\right|}{n}
$$

Input data in the table, the average error value was:

$$
\mu=\frac{0.00+0.25+\cdots+0.25}{11} \mathrm{mil}=0.23 \mathrm{mil}
$$

It can be known that the measurement accuracy of the design system can be guaranteed in the range of $0.25 \mathrm{mil}$.

\section{Summary}

The design finally achieved angle measurement with large-range and high-precision quality. In measuring state, the CPLD decoding program and the MSP430 control program run independently, reasonably adjusting the balance of performance and power of signal decoding. According to the test, measurement accuracy of system could reach $0.25 \mathrm{mil}$, which met the actual measuring requirements, and the design had great value in engineering application.

\section{References}

[1] LIANG changyin. The application of grating sensor in the shift automatic measurement system[J]. Instrument Technique and Sensor, 2001,(3):12-13.

[2] LIU Yangyang. The design of minitype grating movement sensor[D]. Changchun University of Science and Technology. 2009.

[3] ZHENG Liping, YIN Guoliang, YAN Wen, et al. Arc-shaped grating inclination sensor and arc-shaped grating inclination measuring instrument: CN201935693U[P] .2011-08-17.

[4] LIU Zhongli. Research on dividing and sensing about optical grating signal in angle measure unit [D]. Changchun: Changchun University of Science and Technology. 2009.

[5] XIE Kai, ZHAO Jian.MSP430 series MCU system program design and practice[M]. BeiJing: China Mechine Press, 2009. 